\title{
Multi-Mission Synergies in Routine Operations of Low Earth Orbiting Satellites
}

\author{
G. Morfill ${ }^{1}$, E. Maurer ${ }^{2}$, S. Zimmermann ${ }^{3}$ and R. Nibler ${ }^{4}$ \\ German Aerospace Center (DLR), Oberpfaffenhofen, 82234 Wessling, Germany
}

\begin{abstract}
The German Space Operations Center currently operates five low Earth orbit satellites in routine phase. The supported missions are the GRACE-mission (two satellites with an along track separation of $200 \mathrm{~km}$ ), the TerraSAR-X/TanDEM-X mission (two satellites in close formation flight at few hundred meters distance) and the Firebird mission with an infra-red camera on the spacecraft TET-1. The Firebird mission will be extended by a second spacecraft BIROS in near future. Effort has been spent to exploit synergy potentials in operations of these spacecraft. Since 2014 they are controlled in a multi-mission control room to facilitate combined operations for the multi-mission flight support team. The concept of the multi-mission layout of the control room will be described in this paper. Control room activities of low Earth orbiting satellites are driven by ground stations passes. Maximal synergies are possible whenever the ground station passes of the different missions are homogenously distributed over the day. In this case a minimum of multi-mission flight personnel is able to support the different missions sequentially. However, the timing of the ground station passes may not be chosen freely as the visibility times of ground stations are given by the combination of orbit and the geolocation of the available ground stations. In order to avoid conflicts with support times of other satellites a choice between different visibilities in sub sequential orbits is, in some cases, compliant with the mission's operations concept and requirements. Another option might be the selection of alternative ground stations in different parts of the world. The operational integration of new ground stations in the mission's network with an appropriate connection line is a precondition. The missions TerraSAR-X/TanDEM-X and GRACE comprise two spacecraft each. In both cases the spacecraft orbit fly in close spatial proximity and both missions use ground stations of the German Aerospace Center (DLR) in Germany, namely Weilheim in southern part, and Neustrelitz in northern part. One ground station supports the first satellite of the mission, the other ground station the second satellite of the same mission. As a consequence the support times in the control room for the two spacecraft are practically identical. In order to open-up the possibility to support parallel passes with a minimum of staff the operational task during passes needs to be reduced and simplified. This is done by assistance of automatic processes taking care of certain pass preparation functions, commanding and post pass activities or by the restriction of active interaction to one satellite only. The concept ideas are described in the paper. A further complication exists by the fact that the satellites of the GRACE mission do not have a sun synchronous orbit. The passes of the Grace satellites move in daytime in contrast to the ones of the TanDEM-X/TerraSAR-X mission and the TET-1 spacecraft. As a consequence the overall support pattern changes from day to day. An extreme accumulation of support times is possible. In order to avoid extreme situations a multi-mission pass planning process has been initiated.
\end{abstract}

\footnotetext{
${ }^{1}$ TSX/TDX Flight Director, German Space Operations Center, Gary.Morfill@ dlr.de

${ }^{2}$ Group Lead Mission Operations (LEO), German Space Operations Center, Edith.Maurer@dlr.de

${ }^{3}$ TSX/TDX Project System Engineer, German Space Operations Center, Steffen.Zimmermann@ dlr.de

${ }^{4}$ Mission Planning Engineer, German Space Operations Center, Rainer.Nibler@ dlr.de
} 


\title{
Nomenclature
}

\author{
$D L R=$ German Aerospace Center \\ GSOC = German Space Operations Center (part of DLR) \\ OPS = Operations \\ MMFS = Multi Mission Flight Support \\ $F L D=$ Flight Director \\ $F D \quad=$ Flight Dynamics \\ GDS = Ground Data System \\ LEO = Low Earth Orbit \\ $T M \quad=$ Telemetry \\ TC $=$ Telecommand \\ $V M \quad=$ Virtual Machine \\ $O / S \quad=$ Operating System \\ GECCOS = GSOC Enhanced Command- and Control System for Operating Spacecraft \\ $T P C=$ Terminal Personal Computer \\ $O P C=$ Office Personal Computer \\ $D P C=$ Display Personal Computer \\ $P U S \quad=$ Packet Utilisation Standard \\ LTAN = Local Time on Ascending Node
}

\section{Introduction}

$\mathrm{E}$ FFORT has been made at the German Space Operations Center to detect and exploit synergies in routine operations for low Earth orbit satellite missions. In particular GSOC currently operates five LEO satellites in routine phase. The supported missions are the GRACE-mission (Gravity Recovery And Climate Experiment - two satellites with an along track separation of 200km), the TerraSAR-X/TanDEM-X mission (two satellites in close formation flight at few hundred meters distance) and the Firebird mission with an infra-red camera on the spacecraft TET-1 (Technologieerprobungsträger, Technology Experiment Carrier). The Firebird mission will be extended by a second spacecraft BIROS (Bispectral InfraRed Optical System) at the end of May 2016. The goal is to facilitate combined operations for the Operations Team, and especially for the Multi Mission Flight Support Team, and to increase the flexibility and efficiency of the control room environment for multiple satellite missions. The MMFS Team supports the Operations Team in spacecraft control and monitoring by means of a 24/7 shift service.

Therefore a new concept for the multi-mission layout of the control room, in particular the control room consoles, has been established. Furthermore a multi-mission pass planning process has been initiated. In addition automatic processes taking care of certain pass preparation functions, commanding and post pass activities have been introduced.

\section{Fundamentals and traditional Processes}

This chapter points out the motivation for the conceptual modifications and describes the situation before new concepts for multi-mission synergies were developed.

\section{A. Control Room Setup}

Traditionally, each low Earth orbit satellite mission operated at GSOC had a dedicated control room or at least a separate sector in a control room with a fixed number of mission specific consoles. A standard console is a workplace composed of three or four monitors and three computers with different functionalities, different networks and different Operating Systems. Their flexibility is highly limited since the system is configured for one type of task in one dedicated mission with a strict assignment of the $\mathrm{O} / \mathrm{S}$ to certain hardware (see Figure 1). As a consequence the reorganization for another mission is very complicated and time-consuming. 


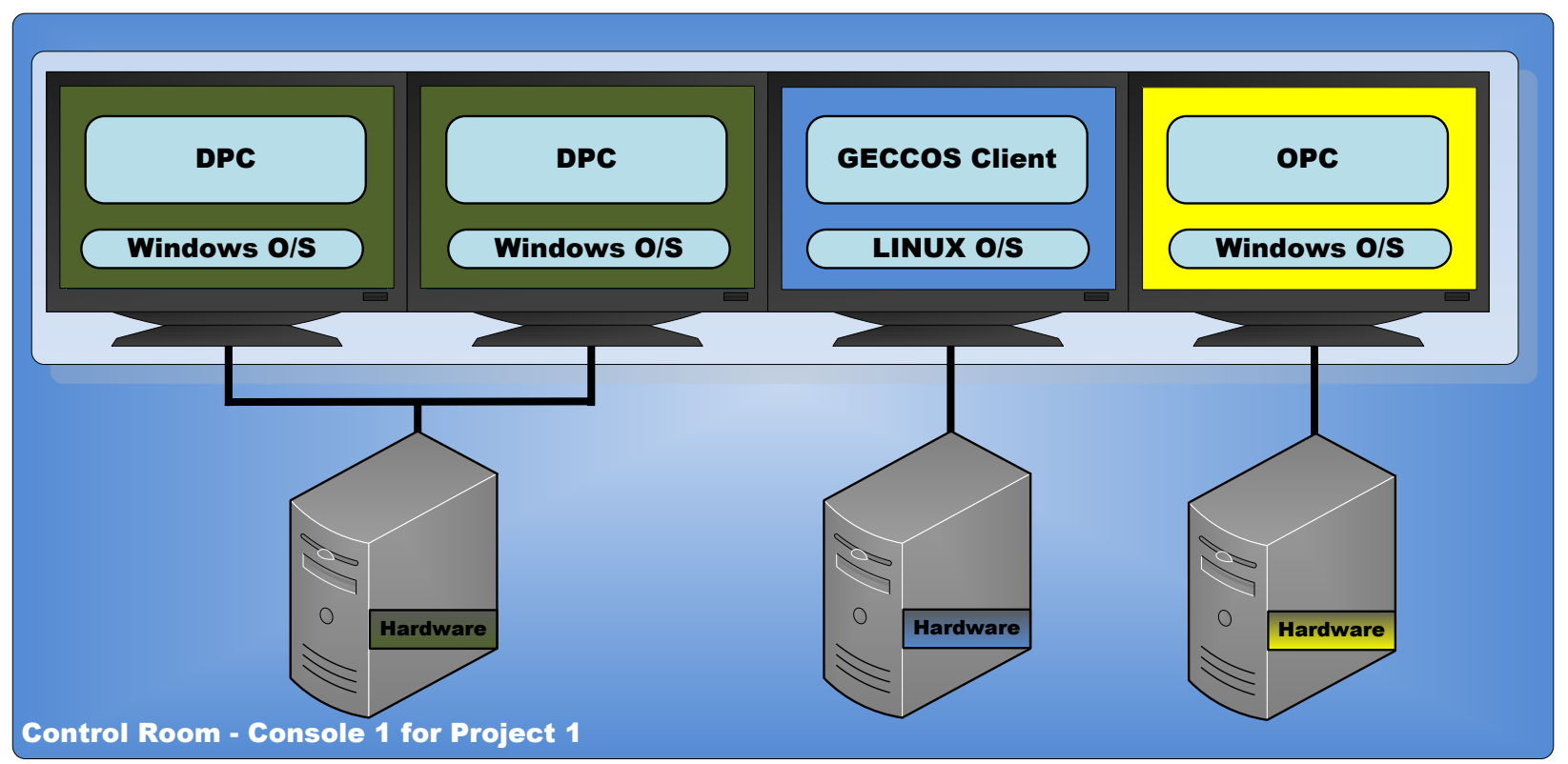

Figure 1. Traditional Control Room Console; DPCs are mainly used for Telemetry Monitoring, GECCOS Clients for the actual commanding or monitoring of the TM/TC chain and the OPCs to gain access to a Web tool for operations, E-mails, or remote connections to the personal office PCs.

Although the various missions differ in a lot of points, the operations workflow and the OPS-Tools used are more or less similar. Therefore it is possible that the OPS team and especially the MMFS team can take care of routine operation tasks for multiple missions. To facilitate this multi-mission support approach, the decision was made to consolidate the increasing number of concurrent missions in one multi-mission control room. In order not to reduce the number of consoles, needed by each mission for safe and reliable operations, a new concept for the control room layout and in particular for more flexible consoles was developed. An easy and fast switch between the different missions at each console is evitable for this new approach. For complex activities or mission phases (e.g. On-Board Software Uploads) a large OPS team with access to the mission consoles is needed for one satellite mission in the control room. The concept and the resulting benefits are described in Chapter III A.

\section{B. Pass Scheduling}

In general the operations activities of LEO satellite missions in routine phase are strongly driven by ground stations passes. Traditionally, each mission manually selected their ground station passes and requested the ground station support times without any interaction and optimization with the other missions operated at GSOC. This pass scheduling workflow, which additionally comprises a lot of costly and manual steps, is shown in Figure 2.

The involved participants are Flight Dynamics, the OPS team, GDS (Ground Data System) Scheduling Office and the ground stations. The Flight Dynamics System offers a Web based interface with the calculated ground station times based on the latest orbit information.

The OPS Team, in particular the Flight Director selects and orders the desired ground station passes via the GDS Scheduling Office, which is responsible for the coordination and communication between the projects and the ground stations. 


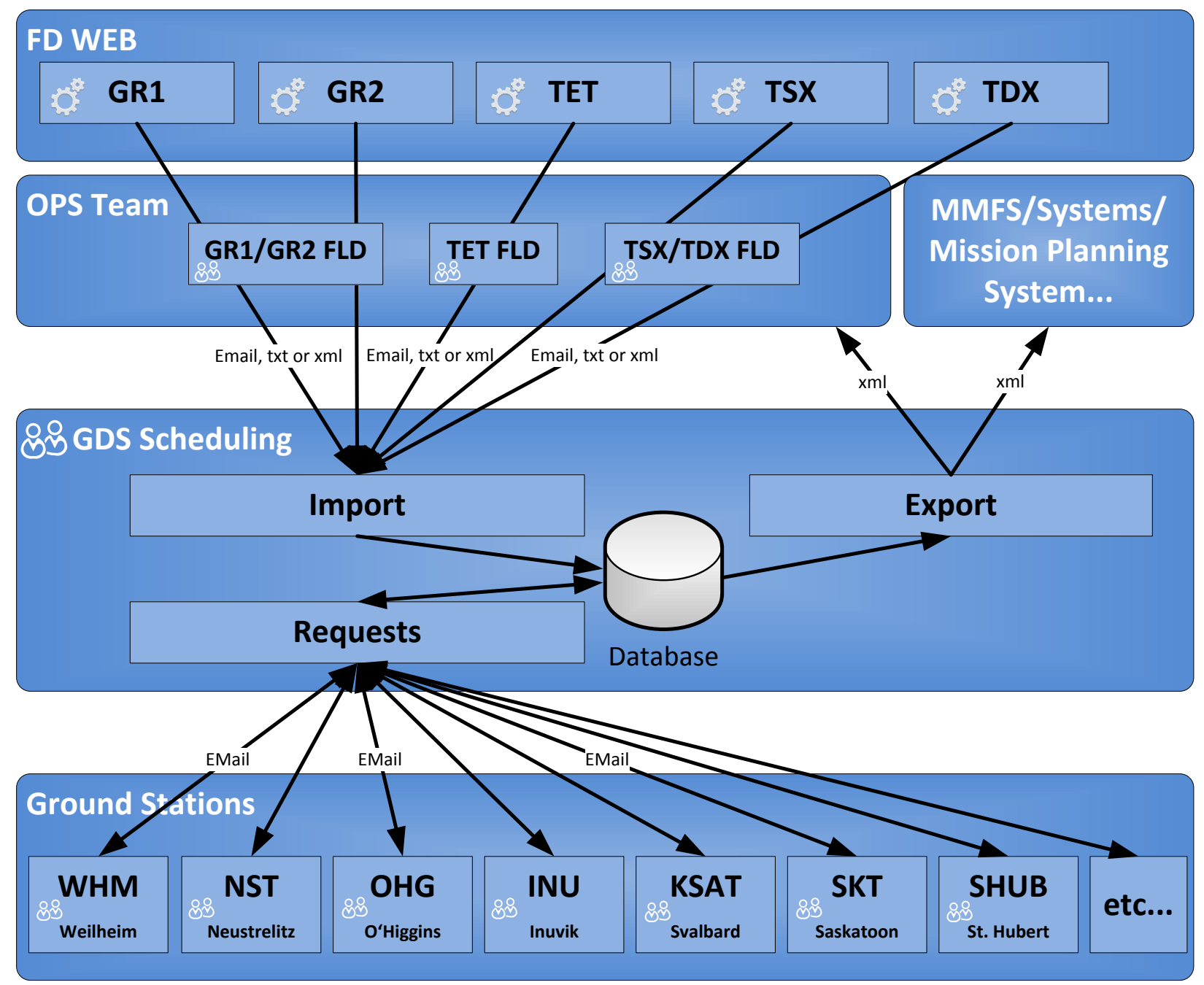

Figure 2. Traditional Pass Scheduling Process; The ground station times can be retrieved via a Web-Service (FD Web). The OPS Team selects and orders the desired ground station passes via the GDS Scheduling Office. Reordering in case of cancellation or special events is done by the OPS team of each project.

Since all LEO missions in routine phase are operated by the MMFS Team, which is available in the control room 24/7, a convenient arrangement of the ground station passes, homogenously distributed over the day, would be beneficial and most helpful for cost efficient operations. In this case a minimum of personnel is able to support the different missions sequentially. Although the pass selection is restricted by certain constraints, a process to optimize the distribution of the passes would be desirable.

However, the timing of the ground station passes may not be chosen freely. The restrictions are given by the visibility times of ground stations, which depend on the combination of orbit and the geolocation of the available ground station antennas, the mission ground station network and other mission constraints, respectively requirements.

In order to achieve a multi-mission pass scheduling process for all LEO satellite missions at GSOC, a new concept and a prototype Tool was developed. This new pass scheduling workflow is described in detail in Chapter III B. 


\section{Commanding, Pre- and Post-Pass Activities}

Furthermore effort was made on the one hand to simplify certain activities and on the other hand to reduce the quantity of work during routine operations for the MMFS team. For LEO satellite missions, operated at GSOC, besides the actual commanding there are a lot of routine activities before and after the ground station contact, which traditionally are manually performed by the MMFS team.

The main routine operations tasks performed by the MMFS are illustrated in the following table.

Table 1. Operator Tasks; This table gives an overview of the tasks, which are traditionally performed by the MMFS team before during and after each pass.

\begin{tabular}{|c|c|}
\hline Type & Task \\
\hline Pre-Pass Operations & $\begin{array}{l}\text { - Check recommendations (formal means by which activities are initiated, } \\
\text { - } \text { reviewed, confirmed and monitored) } \\
\text { - Check completeness of the downlinked Housekeeping-Data } \\
\text { - } \quad \text { Preparation of Command-Stacks with the intended TCs for the upcoming } \\
\text { ground station pass } \\
\text { - Generation of mission specific Products } \\
\text { - } \quad \text { Pre-Pass Briefing with Ground Stations if necessary } \\
\text { other Project specific tasks }\end{array}$ \\
\hline In- Pass Operations & $\begin{array}{l}\text { - Execution of TMConChecks (consisting of a pre-defined set of TM } \\
\text { parameters and values, which are checked against pre-defined ranges) } \\
\text { - Sending of TCs } \\
\text { - Monitoring of TM } \\
\text { - Dump and Clearance of Logs if necessary } \\
\text { - other Project specific tasks }\end{array}$ \\
\hline Post-Pass Operations & $\begin{array}{l}\text { - Consistency Checks e.g. OBQM (On Board Queue Model) } \\
\text { - Close executed Recommendations } \\
\text { - } \text { Fill out console/pass log } \\
\text { - Write E-mails and Anomaly Reports in case of problems } \\
\text { - Check completeness of files generated after each pass (e.g. TC Logs, } \\
\text { OBEH (On Board Event History), TCAH (Telecommand } \\
\text { Acknowledgment History)...) } \\
\text { - Perform clean-up tasks } \\
\text { - other Project specific tasks }\end{array}$ \\
\hline
\end{tabular}

Some of these Activities could be performed by automatic processes or can be shifted in time in order to untangle the concentrated workload around the ground station contacts. The automation of the command chain and other routine operational tasks offers the potential of a substantial cost reduction due to reduced MMFS shifts on console.

From an operational point of view, it is important that an automation system fits in the operational command Workflow, thus ensuring safe and reliable operations. The concept and first implementation of an automation system is described in detail in Chapter III C. 


\section{Technical Approach for the Exploitation of Multi-Mission Synergies}

This chapter describes the technical approach and concepts for the control room setup, the pass scheduling process and the automation of routine operational tasks. The GSOC currently operates five LEO satellites in routine phase. The supported missions are the GRACE mission, the TerraSAR-X/TanDEM-X mission and the Firebird mission with the spacecraft TET-1. In near future more low earth orbit satellites, like BIROS, Eu:CROPIS (Euglena and Combined Regenerative Organic-food Production in Space), GRACE Follow-On and EnMAP (Environmental Mapping and Analysis Program), will be launched and are planned to be integrated into the multi-mission environment.

\section{A. Control Room Setup}

The current five Satellite Missions are controlled in a multi-mission control room since 2014. Therefore a virtual control room concept was developed, implemented and further improved by using modern IT solutions (see ${ }^{[1]}$ ).

This approach has advantages for both operators and administrators. The main objective is the possibility of using control rooms in a much more flexible way. This is especially important because of the increasing number of satellites operated at GSOC in a multi-mission environment. Ideally each control room can be used for all satellite missions involving only minimal reconfiguration effort. This also means that the OPS team will be able to switch quickly and easily between different setups like training- or simulation-sessions using just a single client device. In addition, applications running in external networks can be accessed from the same client device in a secure way. On the one hand, the number of installations can be reduced due to less hardware needed in the control rooms. On the other hand, the maintenance of the whole system will be simplified which should lead to shorter downtimes.

The modernised control rooms at GSOC host standard consoles almost only, this means that all consoles are equipped identically and can in principle be used for all the control room functionalities. So each console is a multimission console and equipped with one shuttle PC and a series of three or four monitors. This shuttle PC is referred to as Terminal PC (TPC) and is used to address the monitors and to connect to virtual machines hosting the control functionalities. The login to the TPC is done via a personal account and the defined projects and corresponding roles are displayed according to the configured user rights. Depending on the selected project and role a desktop layout is displayed with a specific link selection.

The standard links are:

- Link to get a session on a Terminal Server for a virtual Display PC (V-DPC)

- Link to remote connection to a virtual GECCOS client (commanding and monitoring functionality)

- Link to get a Virtual Office PC (V-OPC)

The terminal servers are mission specific and host the display functionalities of the missions (TM monitoring and flight control procedure instantiation). The V-DPC sessions are persistent until explicit log-off, this means, by $\log$-in either a new session is started or the former session is resumed in order to continue the work at the exact same point. For these Applications running on the V-DPC a Windows O/S is needed.

The access to the virtual GECCOS Clients is accomplished by a remote connection via a NX- or X2Go-Client. This connection can be used for the actual commanding, provided that the user has the rights to access this session, or to monitor the TM and TC chain via GECCOS. For this application a Linux O/S is needed.

For the access to a V-OPC it is necessary to pass certain LAN boundaries. On these V-OPCs the users can gain access to the OpsWeb (Web tool for operations), E-mails or open a Remote Desktop connection to their office PCs. This V-OPC uses a Windows $\mathrm{O} / \mathrm{S}$.

The concept and special architecture needed to realize a virtual control room is part of a separate Paper by T. Singer $\left(\right.$ see $\left.^{[3]}\right)$. A simplified overview is shown in Figure 3. 


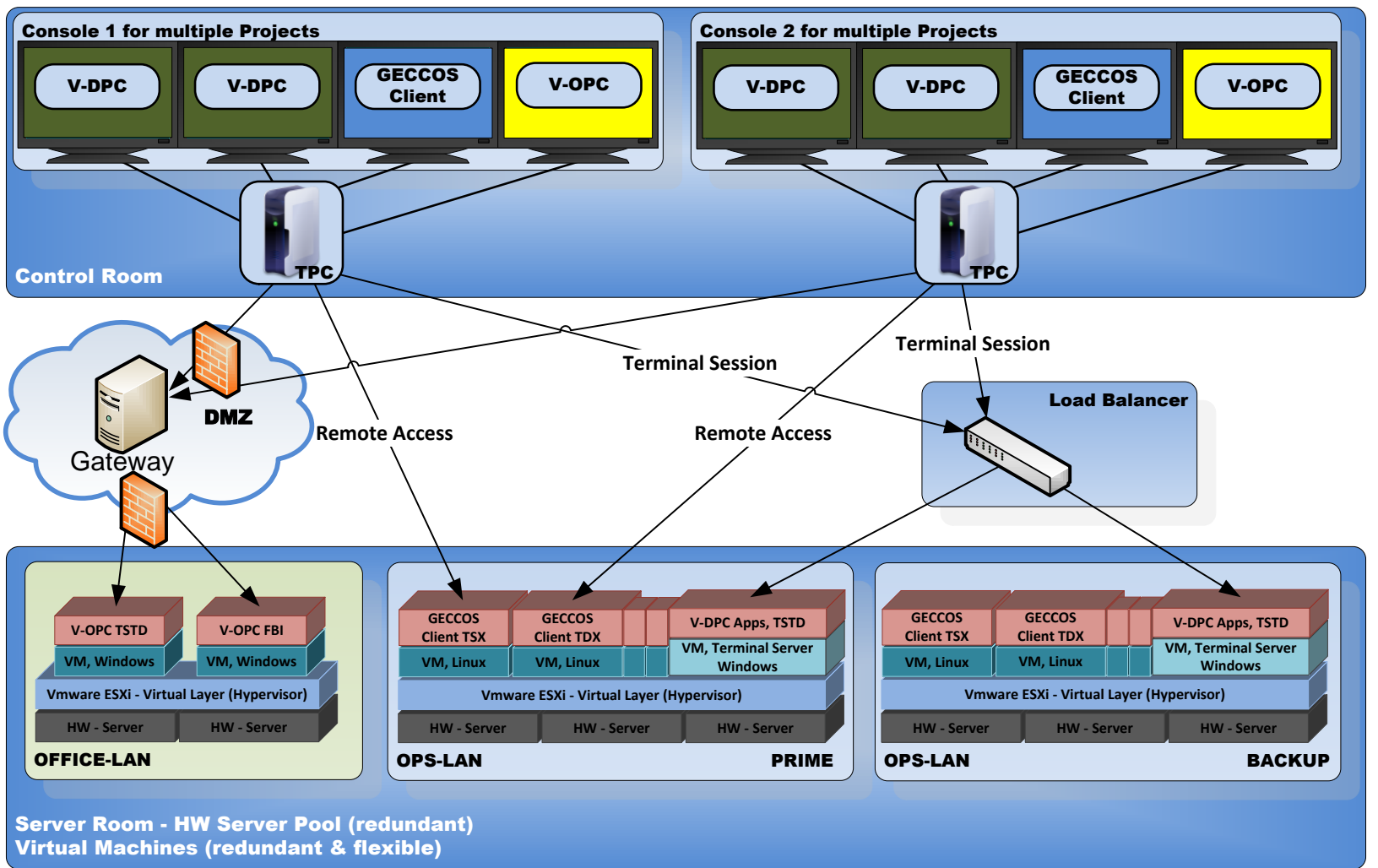

Figure 3. Recent Control Room Setup; This figure illustrates the selected approach to achieve the needed flexibility for a multi-mission control room concept, but does not present a complete overview of the modern control room.

\section{B. Pass Scheduling Process}

A new concept to schedule the ground station passes consolidated for all LEO satellite missions operated at GSOC was developed, with the goal to support the different missions sequentially with a minimum of personnel.

The avoidance of conflicts with support times of other GSOC satellites in some cases can be accomplished by a choice between different visibilities in sub sequential orbits. Another option might be the consideration to introduce alternative ground station antennas in different parts of the world to the mission ground station network. The operational integration of new ground stations in the mission's network with an appropriate connection line is a precondition. So although the potential optimization is limited due to various restrictions, the need for an automated tool for the pass scheduling process was detected.

The possible visibility times of each ground station and satellite configured within the GSOC Flight Dynamics Software are provided to the projects via a File- or Web-Interface. Before this new process, each mission choose the desired ground station contacts according to their ground station network, mission requirements and mission specific needs. These mission specific rules were adapted to algorithms within a multi-mission pass scheduling tool, called MuMiCoRoS (Multi-Mission- and Control-Room-Scheduling). MuMiCoRoS is able to automatically select the contacts for routine operations for the following satellites:

- TerraSAR-X (TSX - dusk-dawn-orbit, LTAN 18:00UTC),

- TanDEM-X (TDX - dusk-dawn-orbit, LTAN 18:00UTC),

- GRACE-1 (GR1 - non-sun-synchronous-orbit, varying LTAN),

- GRACE-2 (GR2 - non-sun-synchronous-orbit, varying LTAN) and the

- $\quad$ TET-1 (TET - sun-synchronous-orbit, LTAN 11:27UTC) 
Furthermore a conflict detection and a simple optimization in terms of the choice between sub-sequential orbits whenever possible takes place. The optimization of course could be enhanced if alternative antennas are integrated into the missions' ground station networks. The designated workflow is shown in Figure 4.

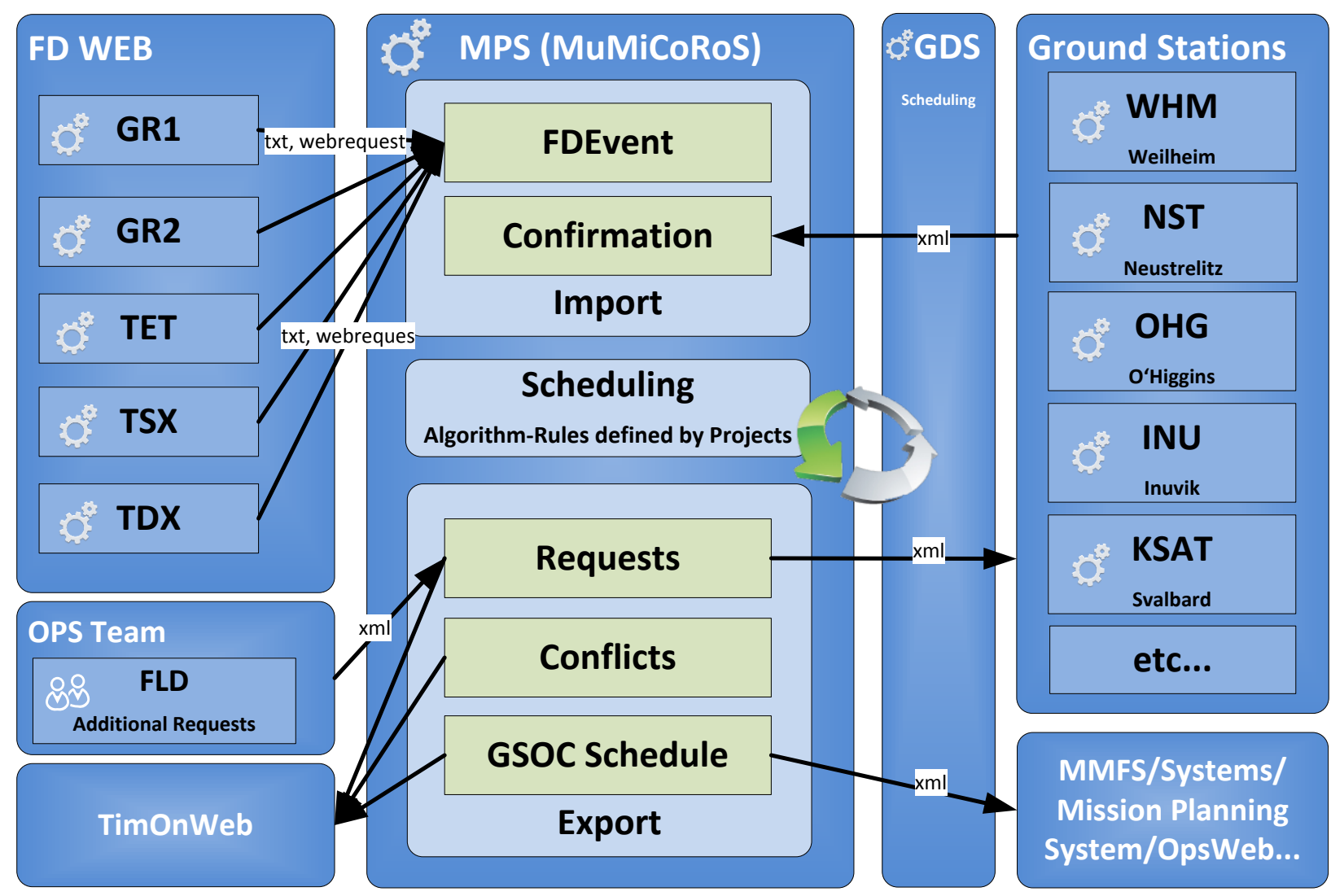

Figure 4. Pass Scheduling Process Improvement; This figure outlines the new workflow for ordering and scheduling of the ground station passes. The elimination of manual interaction and the potential improvement between the concurrent missions was the main motivation for the process enhancement.

\section{Automation of routine operational Tasks}

The TanDEM mission is based on the close formation flight of two radar satellites (TerraSAR-X \& TanDEM-X), both satellites use ground stations of the German Aerospace Center (DLR) in Germany, namely Weilheim in southern part, and Neustrelitz in northern part for the main uplink activities (Mission Timeline generated by the Mission Planning System 1000 TCs twice a day). One ground station supports the first satellite of the mission, the other ground station the second satellite of the same mission. As a consequence the support times in the control room for the two spacecraft are practically identical.

In order to open-up the possibility to support parallel passes with a minimum of staff the operator workload during passes need to be reduced and simplified. Therefore the assistance of automatic processes taking care of certain pass preparation functions, commanding and post pass activities was introduced to the TanDEM mission.

It was decided to use a scripted language as basis for the automation system, which offers the possibility of a simple and flexible implementation. Furthermore an easy integration in an existing monitoring and control system with a well-defined interface, namely the native Test and Operation Procedure Environment (TOPE interface) of the GECCOS (based on SCOS-2000 Release 3) and its dedicated functions already available was advantageous.

GECCOS is the standard monitoring and control system used at GSOC. The automation system is based on several separate scripts, each holding a block of functions for certain basic functionalities that can be adapted to the specific mission needs.

From an operational point of view, it is important that an automation system fits in the operational command Workflow, thus ensuring that parallel manual commanding is still supported and possible. In order to guarantee 
that all uplinked commands were successfully received and executed on-board, it is necessary to explicitly check the TC acknowledgments delivered by the spacecraft. For PUS designed spacecraft the TC acknowledgments offer the possibility to confirm the successful execution or reception on-board in an elegant way via PUS Service 1.

The automation system is able to take care of specific tasks, which can be divided into three main groups:

Table 2. Operations Tasks; This table gives an overview of the tasks, which currently can be performed by the automation process.

\begin{tabular}{|c|c|}
\hline Type & Task \\
\hline $\begin{array}{l}\text { Pre-Pass Operations \& } \\
\text { Cyclic Activities }\end{array}$ & $\begin{array}{l}\text { - System Check } \\
\text { - Preparation of command activities i.e. loading of command sequences to } \\
\text { the MCS system } \\
\text { - Final System Setup }\end{array}$ \\
\hline Pass Operations & $\begin{array}{l}\text { - Detect start of contact (AOS - Acquisition of Signal) } \\
\text { - Check satellite status \& uplink capability via execution of TMConChecks } \\
\text { - Uplink of TCs (Mission Timeline, FD-Manoeuvres...) } \\
\text { - Request of specific TM data dumps } \\
\text { - Detect end of contact (LOS - Loss of Signal) }\end{array}$ \\
\hline Post-Pass Operations & $\begin{array}{l}\text { - Final Uplink integrity check } \\
\text { - Product generation based on real time available from previous contact }\end{array}$ \\
\hline
\end{tabular}

The development and set up of an automation system should take these three operations tasks into account. This means, that not only the in advance prepared TCs are uplinked in an automatic manner, but also certain ground segment related tasks prior and after a real-time contact are subject to the automation system.

Besides the pass related activities performed prior and after a real-time contact certain ground related tasks could be performed automatically as well. Such tasks could be the generation of data products, the clean-up and archiving of procedures or the switch between redundancies at regular intervals. Therefore, a cyclic activity task was installed, which performs any available scripted mission specific task. It is important that these kinds of activities are suspended during a real-time contact and resumed afterwards. Cyclic activities can be categorized according to the needed frequency, e.g. hourly, daily or weekly.

For a real-time contact driven system the most important function - besides the automated uplink - is to autonomously detect the upcoming contact, as this is the base to trigger all following actions. The overview of the automation system is shown in Figure 5. 

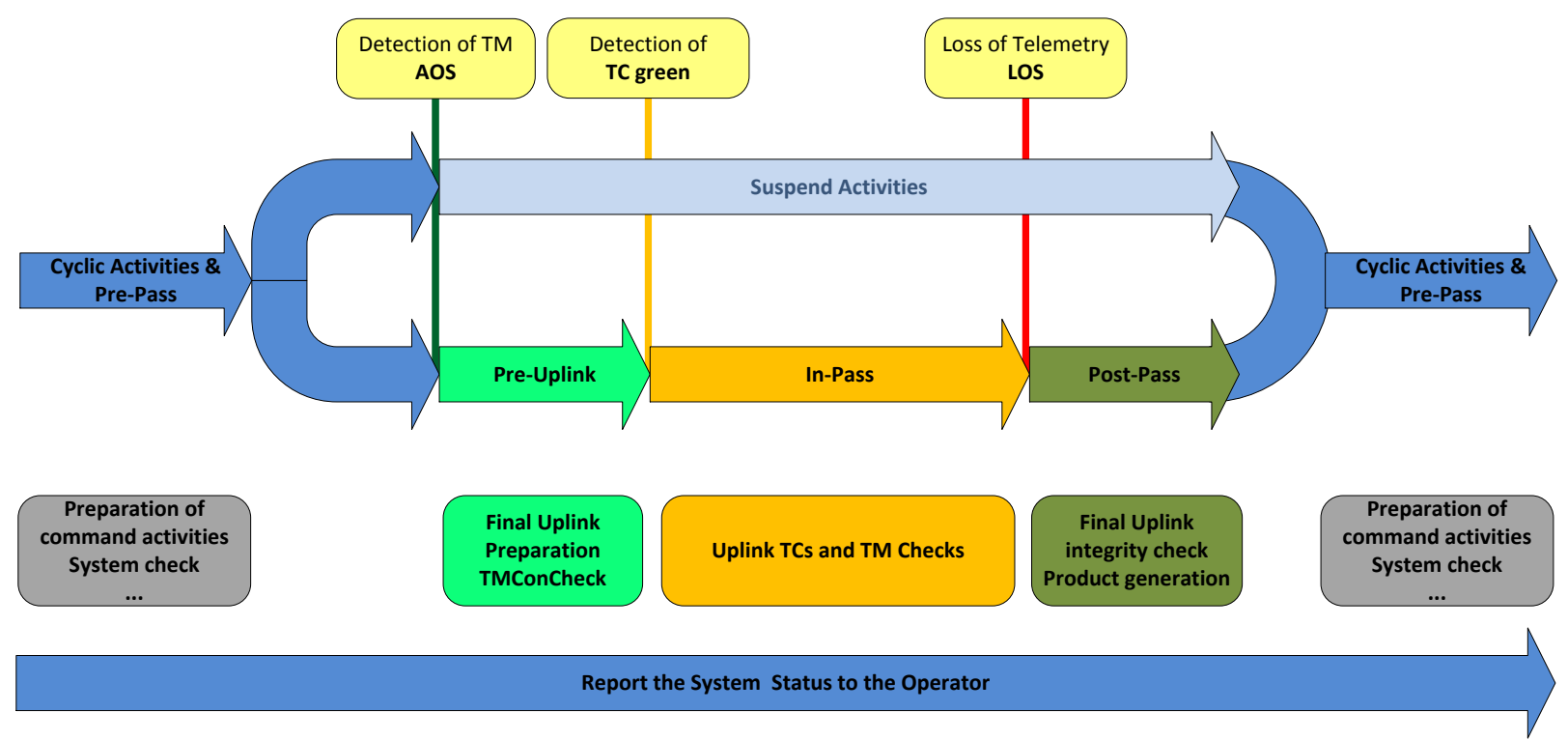

Figure 5. Automation Overview; This figure outlines the rough sequence of the automation process, including the cyclic activities and the activities performed during and directly after a pass.

The detailed concept and special architecture is described in in a separate Paper by S. Zimmermann (see ${ }^{[2]}$ ).

\section{Conclusion and further improvements}

Together, these three enhancements facilitate the integration of multiple satellites into a multi-mission environment and allow cost-efficient operations at GSOC. The new concept for the multi-mission control room at GSOC for all LEO satellite missions in routine phase is also very convenient for all missions in preparation phase. These missions use the control room for test-, training- and simulation-sessions and later on for LEOP- and Commissioning-Phase. So the possibility for the fast and simple switch between concurrent missions is not only applicable for all satellite missions in routine phase, but also helps to avoid timing conflicts between the different preparation activities.

In addition to the already mentioned advantages of the new pass scheduling process, the workflow eliminates a lot of manual and error-prone steps within the former workflow. We plan to have a final easy to adapt implementation of MuMiCoRoS in near feature.

The automation of operational tasks can also be expanded to other missions and new functionalities or tasks can easily be integrated. The first experiences in the routine TanDEM-X mission operations were already gathered, giving a first impression to a future with increased automatic ground operations.

\section{References}

\section{Proceedings} 2012.

${ }^{1}$ Singer, T., Kretschel, U., Schmidhuber, M., "Superior Flexibility for the Control Room Workplace," AIAA, SpaceOPS,

2ZZimmermann, S., Schulze, D., Stangl, C., “Command Chain Automation,” AIAA, SpaceOPS, 2014.

${ }^{3}$ Singer, T., "Implementation of the true Multi-Mission Control Room at GSOC," AIAA, SpaceOPS, 2016. 